\title{
Thermoresponsive hybrid microgel particles with intrinsic optical and magnetic anisotropy $\dagger$
}

\author{
Camille Dagallier, ${ }^{a}$ Hervé Dietsch, ${ }^{* b}$ Peter Schurtenberger ${ }^{b}$ and Frank Scheffold ${ }^{a}$
}

We describe the synthesis of thermoresponsive, magnetic, optically anisotropic and orientable colloidal particles based on poly( $N$-isopropylacrylamide) hybrid microgels (PNIPAMs) with an embedded ellipsoidal hematite $\left(\alpha-\mathrm{Fe}_{2} \mathrm{O}_{3}\right)$ core. Our ability to orient the particles with a magnetic field is demonstrated by small angle X-ray scattering and by optical polarization microscopy.

\section{Introduction}

Stimuli responsive polymers have been extensively developed and studied in recent years, for their potential applications as drug carriers in targeted drug delivery applications, ${ }^{\mathbf{1 , 2}}$ for fundamental studies on self-assembling systems ${ }^{3}$ or on phase behaviour of hard and soft colloidal particles. ${ }^{4,5}$ Among these stimuli responsive polymers, poly( $N$-isopropylacrylamide) (PNIPAM) linear polymers and microgel particles have raised a specific interest for their response to temperature changes., ${ }^{2,4,6}$ Linear chains undergo a coil-to-globule transition when the temperature is increased to approximately $32{ }^{\circ} \mathrm{C}$, and cross-linked PNIPAM particles show an analogous volume phase transition at the volume phase transition temperature $T_{\mathrm{VPT}} \approx 32^{\circ} \mathrm{C}$. The swelling of the microgel particles can be used to tune the volume fraction of particles with an external trigger, ${ }^{8}$ avoiding the classical problems that arise during sample preparation when working with high volume fraction colloidal suspensions, such as high viscosity and aggregation. Moreover, NIPAM can be copolymerized with a variety of co-monomers bearing various functionalities such as acid groups, charges, fluorescent labels, ligands, groups with thermal response or different solubility, chemically reactive and photo-reactive groups, etc. ${ }^{9}$ giving the possibility to further tailor the stimulus sensitivity of microgel particles. In addition core-shell structures with multiple sensitivities can be obtained. ${ }^{7}$ The inclusion of a latex core ${ }^{8}$ or one or several inorganic particles in PNIPAM microgels can also be achieved, leading to further possible applications including catalysis,${ }^{8}$ colloidal crystals, ${ }^{10}$ magnetic carriers, ${ }^{11}$ etc .

Moreover, the study of rotational motion in colloidal systems as a probe of local dynamic properties, such as viscosity or elasticity, has gained attention over the last decade. A large number of studies of this type have been reported in the past using particle tracking on confocal microscopes or depolarized

${ }^{a}$ University of Fribourg, Soft Matter and Photonics Group and Fribourg Center for Nanomaterials, Chemin du muse 3, 1700 Fribourg, Switzerland ${ }^{b}$ University of Fribourg, Adolphe Merkle Institute and Fribourg Center for Nanomaterials, Route de l'ancienne Papeterie, P.O. box 209, 1723 Marly 1, Switzerland. E-mail: herve.dietsch@unifr.ch; Fax: +41 26300 9624; Tel: +41263009137 dynamic light scattering, on geometrically anisotropic systems such as rod-like particles, ${ }^{12,13}$ and on intrinsically optically anisotropic particles, typically colloidal spheres of partially crystalline fluorinated particles. ${ }^{14}$ In soft particle systems, optical anisotropy can be used for example to probe rotational motion and shape fluctuations. Since classical PNIPAM microgel particles are optically isotropic, their rotational motion cannot easily be probed directly. A recent study showed that by including anisotropically distributed nanoparticles in PNIPAM some optical anisotropy of the microgel particles can be obtained. ${ }^{15}$ Here we propose a different approach. We include a spindle-shaped hematite core in the centre of the microgel beads, thus introducing anisotropy in the distribution of refractive index within the particle with the additional advantage that we can orient the particles by means of an external magnetic field. In principle this should allow one to externally control optical properties of microgel based system, although residual optical absorption will be a problem for very dense systems. Using a magnetic core also opens the possibility to study nonlinear rotational dynamics of spherical particles in a visco-elastic matrix. ${ }^{16}$ In addition, optically anisotropic particles are interesting for applications that can exploit externally tuneable properties for optical switching or birefringence. ${ }^{17}$

Here we describe a method developed to synthesize these inorganic-organic hybrid particles (Scheme 1), and demonstrate their potential for the study of rotational motion of particles at high effective volume fractions.

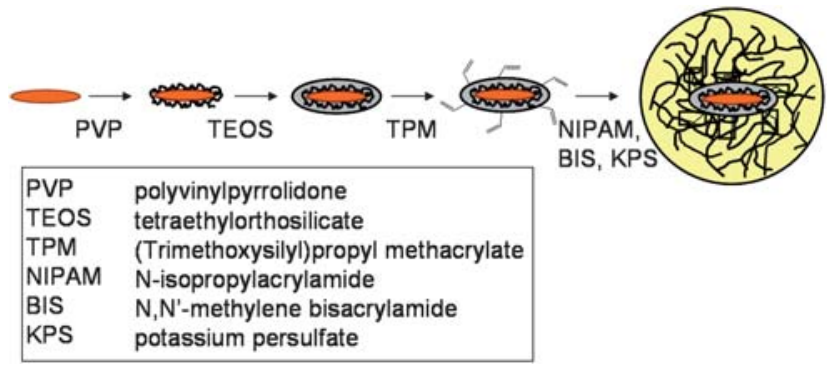

Scheme 1 Illustration of our procedure used to embed hematite spindleshaped particles in PNIPAM microgels. We first add a layer of silica and then modify the surface with reactive silanes allowing the growth of the polymeric shell around the cores. 


\section{Synthesis}

The synthesis of ellipsoidal $\alpha-\mathrm{Fe}_{2} \mathrm{O}_{3}$ (hematite) particles with tuneable size and aspect ratio was reported by Matijevic et al. ${ }^{18}$ and further developed by Ocaña et al. ${ }^{19}$ to reach sub-micrometre scales. The latter method leads to ellipsoidal particles with the long axis corresponding to the $c$-axis of the crystalline lattice. ${ }^{19}$

The particles are coated with a layer of silica using the approach of Graf et al. ${ }^{20}$ based on an initial adsorption of polyvinylpyrrolidone (PVP) on the particles to improve their colloidal stability and the subsequent addition of tetraethylorthosilicate (TEOS) as a precursor for the growth of the silica shell. This method has been found to be more suitable than the original method using bare particles, ${ }^{21}$ as it allows to reach about 6 times higher yields of coated hematite-silica particles when combined with dispersion by ultrasonic waves. ${ }^{12}$

Magnetic PNIPAM microgel particles are traditionally obtained in two steps. Bare microgel particles are first synthesized, and numerous magnetic magnetite or maghemite nanoparticles are subsequently deposited on the surface ${ }^{22,23}$ or synthesized directly in the microgels. ${ }^{11}$ Our approach, however, consists in growing the microgel around the previously prepared magnetic particle. We use the method reported by Zha et al. to grow a PNIPAM microgel around a silica particle. ${ }^{24}$ An analogous method has been shown to be efficient to embed spherical inorganic particles covered with a layer of silica. ${ }^{10}$ To ensure chemical bonding between the inorganic core and the polymeric matrix, the surface of the silica-coated hematite particles is grafted with 3-(trimethoxysilyl)propyl methacrylate (TPM). $N$-Isopropylacrylamide (NIPAM) monomers and a cross-linker, $N, N^{\prime}$-methylene bisacrylamide (BIS) are then polymerized by precipitation polymerization in water in the presence of the surface-modified inorganic particles (Scheme 1).

\section{Results}

\section{Synthesis}

The bare hematite particles used as cores have geometrical dimensions given by the respective average length of the long axis, short axis and aspect ratio - defined as the length of the long axis divided by the length of the short axis - of $300 \pm 40 \mathrm{~nm}$, $56 \pm 6 \mathrm{~nm}$ and $5.5 \pm 0.6$, as measured by transmission electron microscopy. Once adding the thermoresponsive shell, we measure $570 \pm 50 \mathrm{~nm}, 470 \pm 40 \mathrm{~nm}$ and $1.2 \pm 0.2$ for the long axis, the short axis and the aspect ratio respectively. We therefore notice that the aspect ratio is considerably reduced and that the particles are almost spherical in the dried state. When swollen in suspension, we expect this ratio to be even smaller, assuming a homothetic deformation of the microgel shell. We use this assumption in particular when determining the hydrodynamic radius by dynamic light scattering.

\section{Small angle $X$-ray scattering}

Over the range of temperatures used in our experiments, hematite particles are weak canted antiferromagnets with a magnetic moment normal to the long axis (c-axis) ${ }^{25}$ However, for the particles shown in Fig. 1 the cross-linked polymer layer around the hematite spindles acts as a steric barrier. This results in

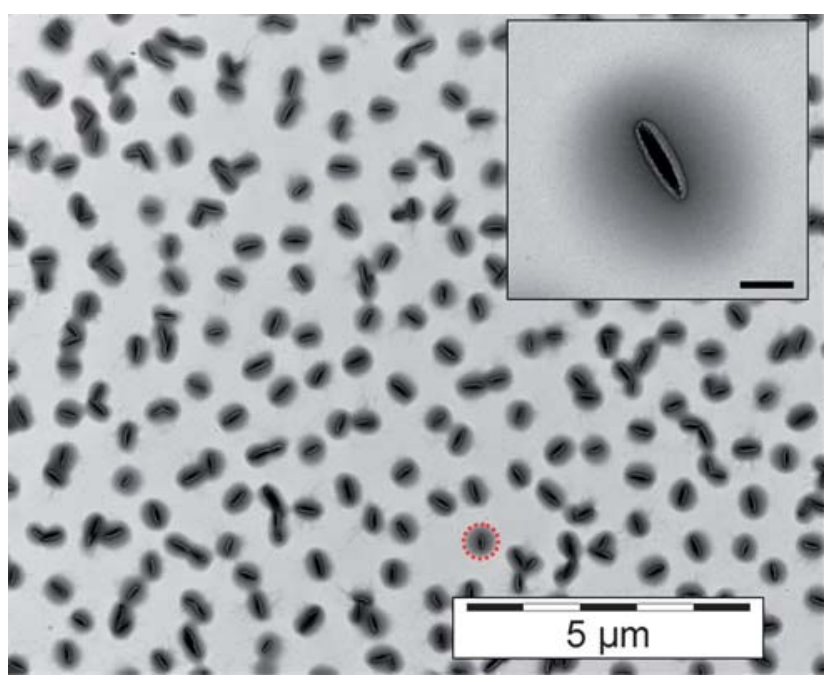

Fig. 1 TEM image of hematite-PNIPAM core-shell particles, a picture of a higher magnification is presented in the inset (scale bar: $200 \mathrm{~nm}$ ) showing a microgel with a size of about $550 \mathrm{~nm}$ in diameter (dried state). The ellipsoidal hematite core and the silica shell are clearly visible due to their different electron contrast. The red dotted circle in the main panel illustrates the estimated size.

a sufficiently large distance between the magnetic cores where interparticle dipole-dipole interactions become negligible at not too high values of an applied magnetic field. With respect to the effective interaction potential between the particles, the microgels thus behave like isotropic hard spheres and the orientation of each particle is therefore decoupled from the orientation of its neighboring particles.

On the other hand we can use their magnetic response to an external magnetic field in order to orient the anisotropic cores

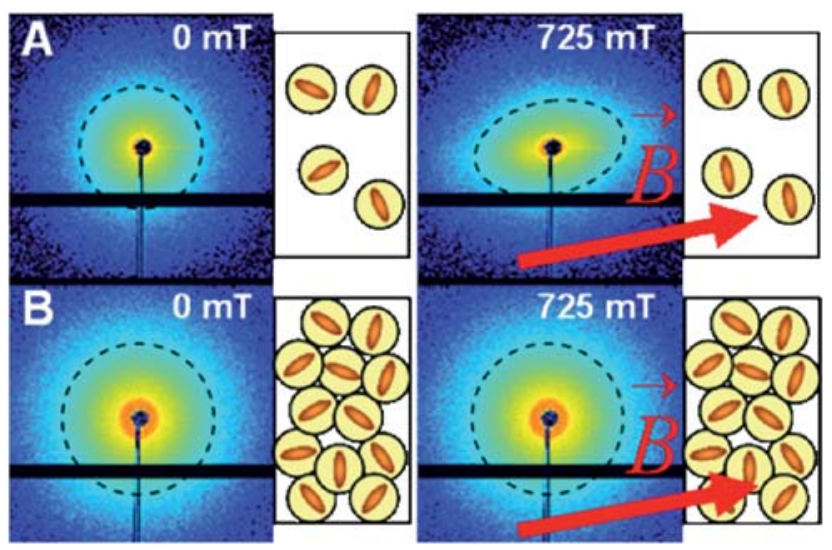

Fig. 2 SAXS scattering pattern measured at $24{ }^{\circ} \mathrm{C}$ without magnetic field and with a $725 \mathrm{mT}$ magnetic field for (A) freely rotating particles (effective volume fraction $\varphi_{\text {eff }}=0.27$ ) and (B) dynamically arrested particles (effective volume fraction $\varphi_{\text {eff }}=0.8$ ). The samples were both prepared initially in the absence of a magnetic field. Dashed lines are guides to the eye. Corresponding orientation of particles is schematically represented next to each pattern. Red arrows show the direction of the magnetic field. Effective volume fractions $\varphi_{\text {eff }}$ are estimated from number densities $n_{\text {conf }}$ measured on a confocal microscope and hydrodynamic radii $r_{\mathrm{h}}$ measured by dynamic light scattering using $\varphi_{\text {eff }}=4 / 3 \pi n_{\text {conf }} r_{\mathrm{h}}{ }^{3}$. 
along a preferred direction. This alignment is confirmed by the anisotropic scattering patterns obtained by small angle X-ray scattering in a liquid suspension of PNIPAM-hematite hybrid microgels (Fig. 2A). Without magnetic field the scattering pattern is isotropic, confirming the random orientation of the cores. However, when the suspension is subjected to a magnetic field we observe an elliptical scattering pattern. This clearly indicates a preferred orientation of the particles, with the long axis perpendicular to the magnetic field B. Moreover after removing the magnetic field the scattering pattern relaxes to its initial isotropic shape. The absence of memory effect shows that magnetic interactions between the cores are indeed negligible.

It has previously been demonstrated that microgels can undergo a hard sphere glass transition around a volume fraction $\Phi_{\mathrm{g}}$ corresponding to random close packing $\left(\Phi_{\text {rcp }} \approx 0.64\right)$ for temperatures at or below $T_{\mathrm{VPT}}$. We thus perform the same experiment using a more concentrated dispersion such that the increase of the effective volume fraction at lower temperatures results in an arrested solid phase that corresponds to a so-called squeezed state. ${ }^{4,6,26}$ In this case the application of a magnetic field does not affect the isotropy of the scattering pattern (Fig. 2B), which shows that the rotation of particles is inhibited at this high effective volume fraction.

\section{Optical polarization microscopy}

As previously demonstrated we can explore the thermal response of the PNIPAM shell surrounding the magnetic core in order to tune the effective volume fraction and reversibly cycle from a dynamically arrested state to a liquid-like state where free rotational motion is restored. Moreover, when combining this with an externally applied magnetic field we can align the magnetic cores in the liquid state prior to cooling the sample. Given the intrinsic optical anisotropy of our hybrid particles, the distribution of refractive indices in the sample thus becomes macroscopically anisotropic and the sample will be birefringent. Quite analogous to suspensions of anisotropic colloidal particles where the alignment of the particles in a liquid crystalline phase induces birefringence we thus expect that we can investigate the macroscopic orientation of the ellipsoidal magnetic cores using optical polarization microscopy. ${ }^{27}$

We use a polarizing microscope equipped with a heating stage to study the evolution of the alignment of the particles as a function of temperature between $10{ }^{\circ} \mathrm{C}$ and $34{ }^{\circ} \mathrm{C}$. In a pretreatment step we align the particles with an external magnetic field at $35^{\circ} \mathrm{C}$, where the sample exhibits liquid-like behavior, and subsequently quench the sample for ten minutes in this aligned state to a low temperature, $10{ }^{\circ} \mathrm{C}$, where the sample is dynamically arrested. After removing the magnetic field, we increase the temperature in steps of three minutes each and record the transmitted intensity $I_{\mathrm{VH}}$ for crossed polarizers. We average the value over the last minute. The same procedure is repeated without prior magnetic alignment of the particles, and these measurements serve as a baseline $I_{0}$ for our analysis. These reference measurements are crucial as the high density of our particle dispersion combined with the temperature-dependent scattering contrast of the microgel shell results in a variable amount of multiple scattering that causes some degree of depolarization even for randomly orientated particles.
We subtract this baseline contribution and obtain a direct experimental quantification $\Delta I=I_{\mathrm{VH}}-I_{0}$ for the degree of alignment of the magnetic cores. As we increase the temperature $\Delta I$ decreases (Fig. 3), indicating that the alignment is progressively lost when the samples go from a jammed arrested state to a liquid-like state.

At the solid to liquid transition $\Delta I$ drops to zero, indicating that the random orientation of the particle cores is recovered. This behavior again confirms that there is no magnetic memory effect, supporting the assumption that magnetic dipole-dipole interactions are negligible. The transition occurs around $27^{\circ} \mathrm{C}$, which corresponds to an effective volume fraction of approximately 0.6 . Free rotational motion is thus restored at an effective volume fraction that seems to qualitatively coincide with the previously reported glass transition where long-range translational motion becomes arrested. Given the previously postulated subtle differences between the dynamics of classical hard sphere colloids and microgels at and beyond the arrest transition, ${ }^{26}$ it will be interesting to quantitatively compare the arrest transition for translational and rotational diffusion using our particles. This will allow us to critically re-examine the importance of the classical cage picture for the arrest transition in particles with a partially soft and interpenetrable shell.
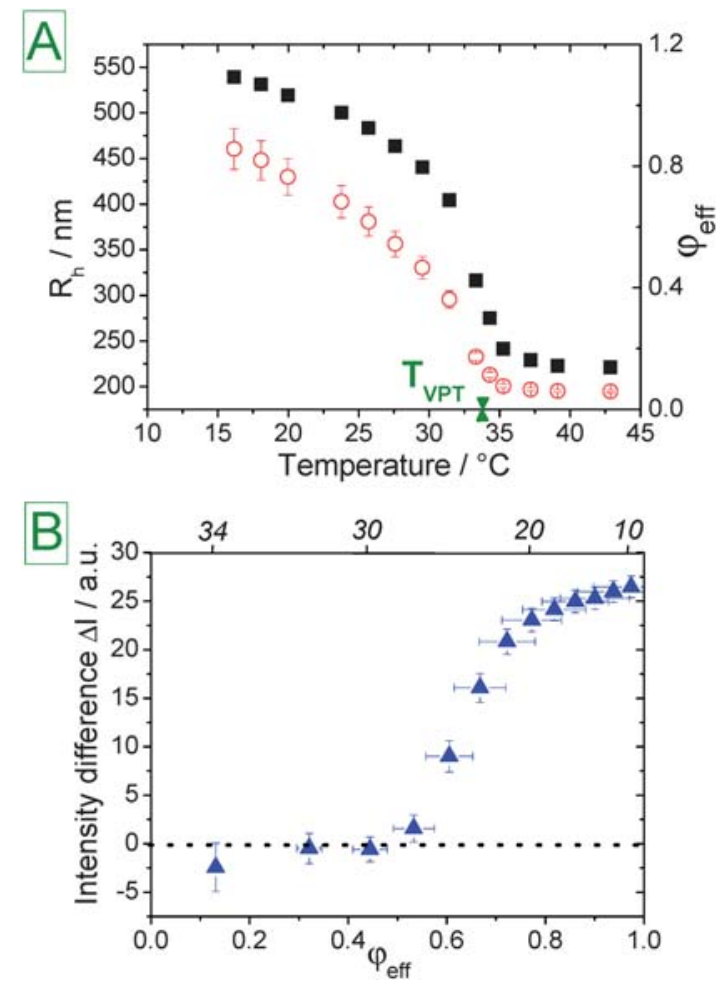

Fig. 3 (A) Black filled squares: hydrodynamic radius of the hematitePNIPAM core-shell particles measured by dynamic light scattering (DLS). Red open circles: effective volume fraction $\varphi_{\text {eff }}$ deduced from DLS data and confocal microscopy. (B) Intensity difference $\Delta I=I_{\mathrm{VH}}-I_{0}$ as a function of effective volume fraction determined by optical polarization microscopy as a measure of the optical anisotropy of the sample. As a support, corresponding temperatures (in degree centigrade) have been indicated on the upper $\mathrm{X}$-axis. 


\section{Conclusion}

In summary we presented for the first time a route to obtain orientable magnetic and thermoresponsive spherical particles with intrinsic anisotropy. These hybrid core-shell particles combine the temperature sensitivity of PNIPAM microgels with the anisotropic and magnetic features of hematite ellipsoids. The possibility to orient particles in the liquid state and the absence of rotational motion at high volume fractions could be demonstrated by small angle X-ray scattering and optical polarization microscopy. Considering the major improvement in terms of control and characterization of rotational motion that the addition of ellipsoidal hematite cores to PNIPAM microgels has led to, this new hybrid system is an ideal candidate for further fundamental studies on model colloids. Combined with the possibility to add functionalities by copolymerization of NIPAM with various monomers, it also has a remarkable potential for different applications involving its magnetic and thermoresponsive properties, for example as controllable tracer particles, in the domain of targeted drug delivery, or as building blocks for stimuli responsive optically anisotropic materials.

\section{Acknowledgements}

The small angle X-ray scattering patterns were measured at the cSAXS beam line of the Swiss Light Source at the Paul Scherrer Institute (Villigen, Switzerland), the authors thank in particular A. Menzel for his assistance during the measurements and M. Reufer for his help with the initial data treatment. J.-F. Dechezelles is thanked for his help with the design of the cover picture. We also would like to acknowledge the financial support for the project provided by the Adolphe Merkle Foundation and the Swiss National Science Foundation (project No. 200020-117762/126772/117755).

\section{Notes and references}

1 E. S. Gil and S. A. Hudson, Prog. Polym. Sci., 2004, 29, 1173.

2 A. Chilkoti, M. R. Dreher, D. E. Meyer and D. Raucher, Adv. Drug Delivery Rev., 2002, 54, 613.
3 J. Rodriguez-Hernandez, F. Checot, Y. Gnanou and S. Lecommandoux, Prog. Polym. Sci., 2005, 30, 691.

4 H. Senff and W. Richtering, J. Chem. Phys., 1999, 111, 1705.

5 U. Gasser, B. Sierra-Martin and A. Fernandez-Nieves, Phys. Rev. E: Stat. Phys., Plasmas, Fluids, Relat. Interdiscip. Top., 2009, 79, 051403.

6 M. Reufer, P. Diaz-Leyva, I. Lynch and F. Scheffold, Eur. Phys. J. E, 2009, 28, 165.

7 C. D. Jones and L. A. Lyon, Macromolecules, 2000, 33, 8301.

8 M. Ballauff and Y. Lu, Polymer, 2007, 48, 1815.

9 S. Dai, P. Ravi and K. C. Tam, Soft Matter, 2009, 5, 2513.

10 M. Karg, I. Pastoriza-Santos, L. M. Liz-Marzan and T. Hellweg, ChemPhysChem, 2006, 7, 2298.

11 D. Suzuki and H. Kawaguchi, Colloid Polym. Sci., 2006, 284, 1443.

12 S. Sacanna, L. Rossi, B. W. M. Kuipers and A. P. Philipse, Langmuir, 2006, 22, 1822.

13 D. Mukhija and M. J. Solomon, J. Colloid Interface Sci., 2007, 314, 98.

14 V. Degiorgio, R. Piazza and T. Bellini, Adv. Colloid Interface Sci., 1994, 48, 61 .

15 S. Bolisetty, M. Hoffmann, S. Lekkala, T. Hellweg, M. Ballauff and L. Harnau, Macromolecules, 2009, 42, 1264.

16 N. Cappallo, C. Lapointe, D. H. Reich and R. L. Leheny, Phys. Rev. E: Stat. Phys., Plasmas, Fluids, Relat. Interdiscip. Top., 2007, 76, 031505.

17 D. R. Cairns, M. Sibulkin and G. P. Crawford, Appl. Phys. Lett., 2001, 78, 2643.

18 E. Matijevic and P. Scheiner, J. Colloid Interface Sci., 1978, 63, 509.

19 M. Ocaña, M. P. Morales and C. J. Serna, J. Colloid Interface Sci., 1999, 212, 317.

20 C. Graf, D. L. J. Vossen, A. Imhof and A. van Blaaderen, Langmuir, 2003, 19, 6693.

21 M. Ohmori and E. Matijevic, J. Colloid Interface Sci., 1992, 150, 594.

22 J. E. Wong, A. K. Gaharwar, D. Muller-Schulte, D. Bahadur and W. Richtering, J. Colloid Interface Sci., 2008, 324, 47.

23 J. Rubio-Retama, N. E. Zafeiropoulos, C. Serafinelli, R. RojasReyna, B. Voit, E. L. Cabarcos and M. Stamm, Langmuir, 2007, 23, 10280 .

24 L. S. Zha, Y. Zhang, W. L. Yang and S. K. Fu, Adv. Mater., 2002, 14, 1090.

25 M. Reufer, H. Dietsch, U. Gasser, A. Hirt, A. Menzel and P. Schurtenberger, J. Phys. Chem., 2010, accepted; D. J. Dunlop, Ann. Geophys., 1971, 27, 269.

26 D. A. Sessoms, I. Bischofberger, L. Cipelletti and V. Trappe, Philos. Trans. R. Soc. London, Ser. A, 2009, 367, 5013.

27 J. C. P. Gabriel, C. Sanchez and P. Davidson, J. Phys. Chem., 1996, 100, 11139; C. Ozdilek, E. Mendes and S. J. Picken, Polymer, 2006, 47, 2189. 


\section{Supporting information.}

\section{Synthesis}

In all the syntheses, water refers to MilliQ water (Resistivity $18.2 \mathrm{M} \Omega . \mathrm{cm}$ ).

\section{Hematite ellipsoids}

The synthesis of ellipsoidal hematite particles is based on a method described by Ocaña et. al ${ }^{l}$, consisting in precipitating the iron oxide in the presence of urea and a phosphate salt. In a typical synthesis, an aqueous solution of iron (III) perchlorate $(100 \mathrm{mM}$, Alpha Aesar, reagent grade), sodium phosphate monobasic $(5.5 \mathrm{mM}$, Fluka, $>99 \%)$ and urea $(100 \mathrm{mM}$, Fluka, $>99.5 \%$ ) was aged at $98^{\circ} \mathrm{C}$ for 24 hours. The precipitated particles (figure S1) were then washed five times by centrifugation followed by redispersion in water.

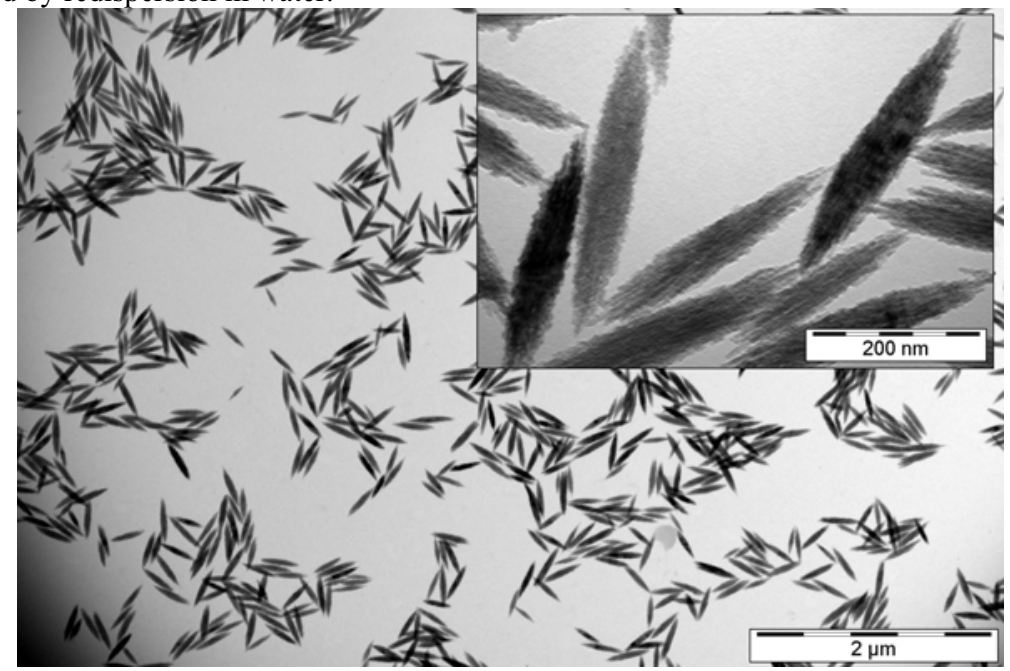

Figure S1. Transmission Electron Microscopy picture of ellipsoidal hematite $\left(\alpha-\mathrm{Fe}_{2} \mathrm{O}_{3}\right)$ particles obtained by precipitation of the iron oxide in the presence of urea and phosphate salt.

\section{Coating with Silica}

Following the approach of Sacanna et. $a l^{2}$, we dispersed $150 \mathrm{mg}$ of particles in a solution of polyvinylpyrrolidone (Sigma Aldrich, $\mathrm{M}_{\mathrm{w}} 40000 \mathrm{~g} \cdot \mathrm{mol}^{-1}, 100 \mathrm{~g} / \mathrm{mL}$ ). The chosen amount of PVP corresponds to about 13 PVP molecules per square nanometer of particle surface. The suspension was stirred for 12 hours to let the polymer adsorb onto the surface. The stabilized particles were then transferred in $485 \mathrm{~mL}$ of a mixture of water $(17.5 \%)$, absolute ethanol $(82.5 \%)$ and tetramethylammonium ( $0.015 \%$, Sigma Aldrich, $25 \%$ solution in methanol). While stirring and sonicating, a $1: 2$ mixture of tetraethylorthosilicate (Aldrich, 99.0\%) and ethanol was added in three shots of $6 \mathrm{~mL}$ each every 20 minutes. After the last injection, the suspension was stirred for 12 hours. The coated particles were then washed 3 times in absolute ethanol and heated up to $60^{\circ} \mathrm{C}$. After injecting $80 \mu \mathrm{L}$ of 3-Methacryloxypropyltrimethoxysilane (ABCR, $>98 \%$ ), the suspension was vigorously stirred for 8 hours, keeping the temperature constant. The particles were then washed by centrifugation and redispersion.

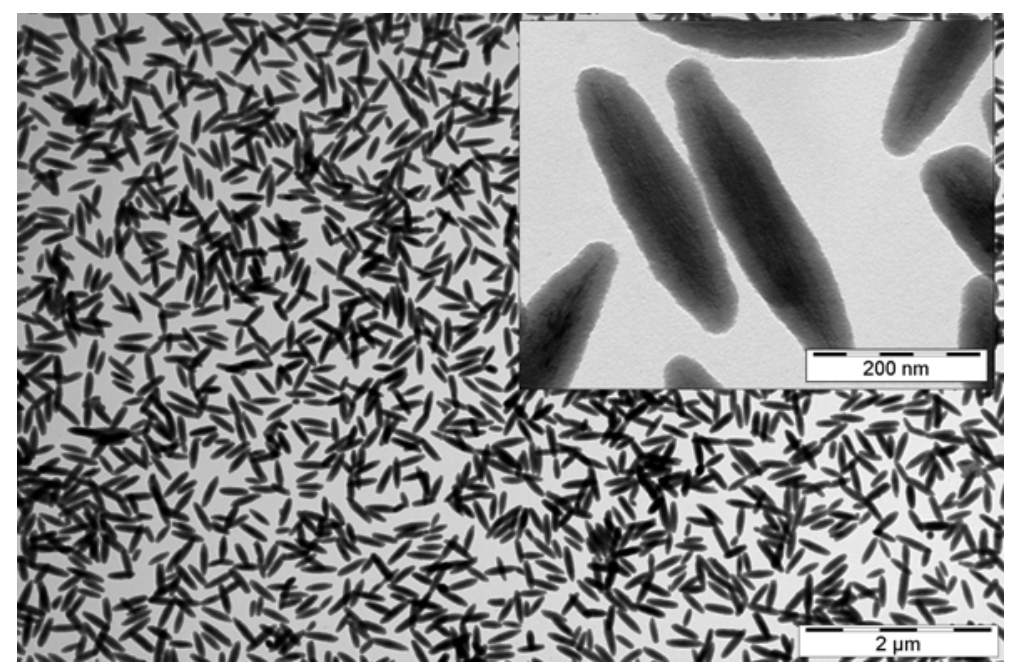

Figure S2. Transmission Electron Microscopy picture of ellipsoidal hematite particles coated with a layer of silica. 
Supplementary Material (ESI) for Soft Matter

This journal is (c) The Royal Society of Chemistry 2010

\section{PNIPAM microgel shell}

Inorganic-organic hybrid particles were obtained by free-radical polymerization of NIPAM in the presence of crosslinking monomers and the silica coated hematite particles. The suspension of surface-functionalized hematite-silica particles was added to an aqueous solution of $\mathrm{N}$-isopropylacrylamide $(60 \mathrm{mM}$, Acros Organics, $99 \%$, recrystallized three times in hexane), N,N'- methylenebisacrylamide (3.4mM, Sigma Aldrich, $99 \%$ ), heated up to $70^{\circ} \mathrm{C}$ and bubbled with Argon for 30 minutes. A degassed solution of potassium persulfate (Fluka, $>99 \%)$ was injected $(3.15 \mathrm{mM}$ in the reaction medium just after injection). The solution was stirred for 4 hours. The particles were then separated and washed by multiple centrifugation steps and finally redispersed in water to reach the desired volume fraction.

\section{Dynamic light scattering and effective volume fraction}

The measurement of the hydrodynamic radius at different temperatures was conducted on a setup based on an ALV/DLS/SLS-5000F monomode fiber compact goniometer system. The average particle diffusion coefficient was obtained from a fit to measured $\mathrm{q}$-dependent relaxation time $\tau=1 /\left(\mathrm{Dq}^{2}\right)$, where $\mathrm{q}$ denotes the scattering wave number. The particle radius then follows from Stokes-Einstein equation. Due to the onset of water condensation the temperature range was limited to $\mathrm{T}>16^{\circ} \mathrm{C}$. We extrapolated the data to lower temperatures by using a divergence fit $\mathrm{R}_{\mathrm{h}}=\mathrm{A}(1-\mathrm{T} / \Theta)^{\mathrm{n}}$ where $\Theta$ is the divergence temperature and $A$ and $\alpha$ are empirical parameters. The fit is shown in figure S5.

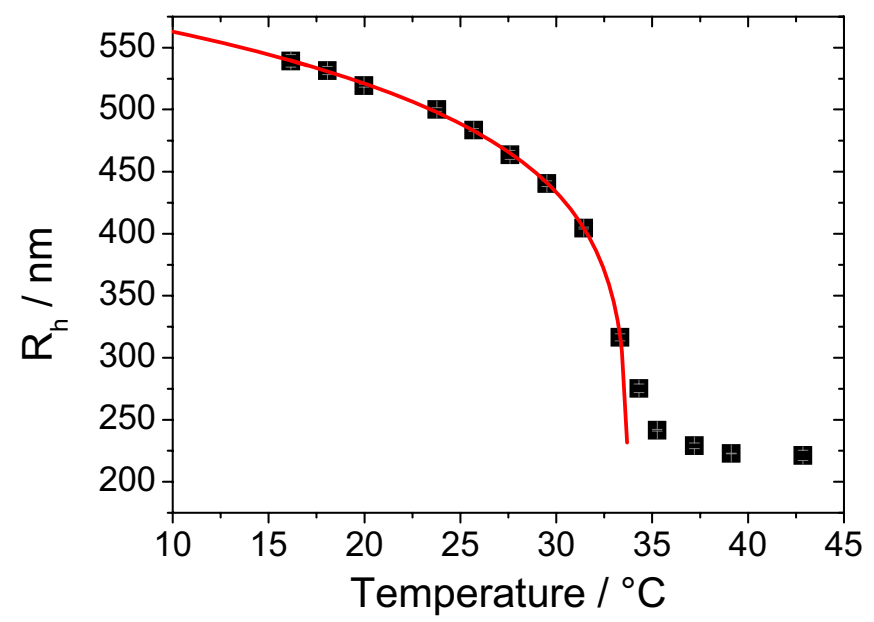

Figure S3. Hydrodynamic radius measured by dynamic light scattering as a function of temperature. Error bars were obtained on the basis of three sets of experiment. The red line corresponds to the fit $R_{h}=A(1-T / \Theta)^{0.17}$ with $A=591.6 \mathrm{~nm}, \Theta=33.7 \mathrm{~K}$.

The particle number density $\mathrm{n}_{\text {part }}$ was determined with a Leica TCS SP5 laser scanning confocal microscope. Prior to the measurement the suspension was diluted with glycerol to slow down Brownian motion and to avoid overlapping particle images.

\section{SAXS data}

To study the particle alignment we analyzed the magnetic field dependence of the 2D SAXS pattern recorded at the Swiss Light Source (Paul Scherrer Institute, Villingen, Switzerland). We subdivided the pattern in sections of $5^{\circ}$ and integrated the scattered intensity I(q) at any given absolute value of the wavevector q. In Figure S5 we plotted the results of this analysis for the sections parallel $\left(I_{\|}\right)$and perpendicular $(\perp \perp)$ to the magnetic field $(B)$. We found that if the particles had been aligned in the field prior to the measurement the scattered intensity was increased along the field-axis. This observation showed that the particles had a privileged orientation perpendicular to the magnetic field. In contrast, for randomly oriented particles, the scattering pattern was isotropic. 


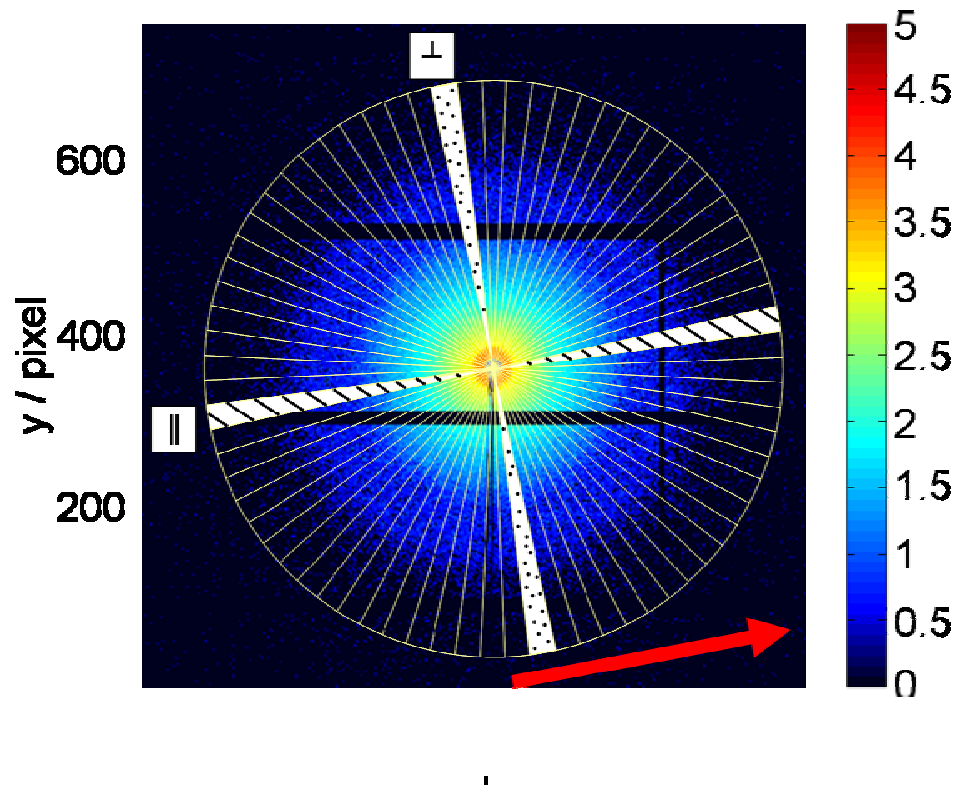

Figure S4. Subdivision of the SAXS scattering pattern in steps of $5^{\circ}$. The perpendicular $(\perp$, dotted) and parallel $(\|$, hatched) directions with regard to the magnetic field are highlighted. Conversion factor pixel-wavevector: $0.001064 \mathrm{~nm}^{-1} / \mathrm{pixel}$.
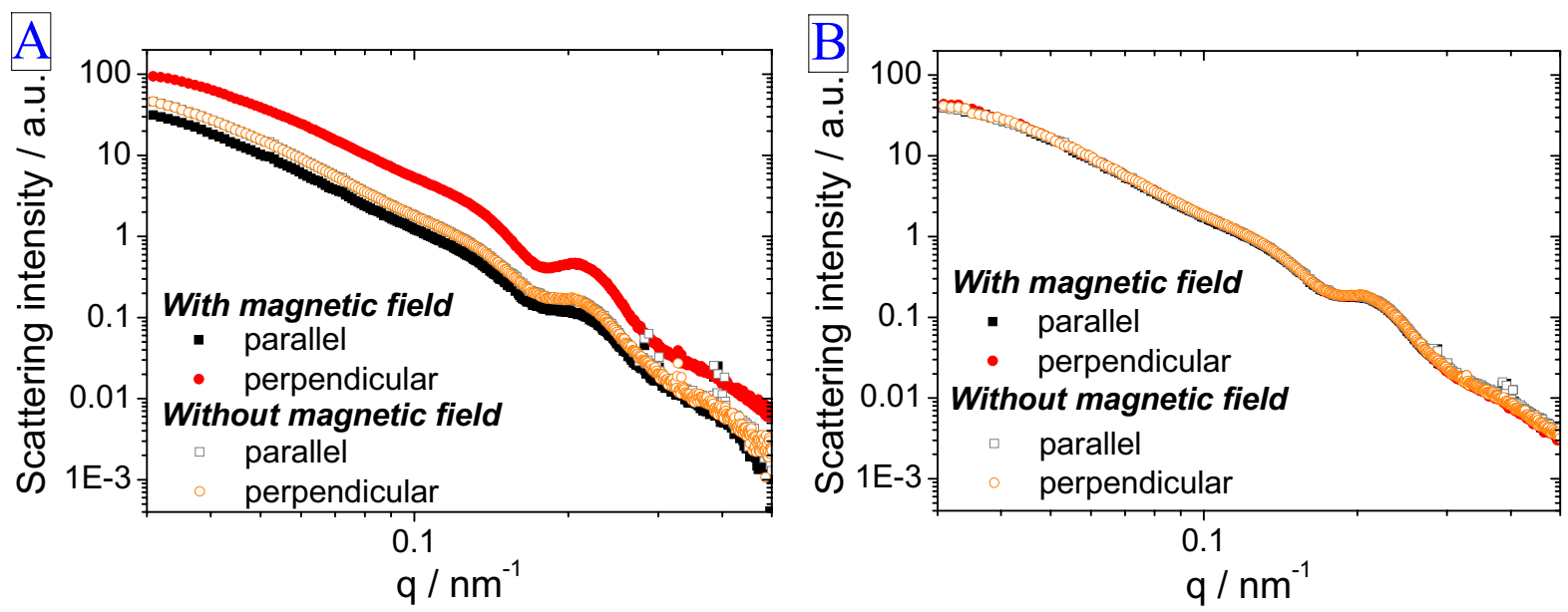

Figure S5. Small-angle X-ray scattered intensity as a function of wavenumber q for different orientations with respect to the field axes. The data shown has been obtained with and without magnetic field $(725 \mathrm{mT})$ for the samples containing rotating particles (A) and dynamically arrested particles (B). The background has been subtracted for both samples. The intensity data shown has been density-adjusted, taking sample (B) as the reference. In the case of dynamically arrested particles, the curves with and without magnetic field superimpose, while in the case of freely rotating particles, the application of a magnetic field leads to an increase in intensity in the direction parallel to the magnetic field and a decrease in the perpendicular direction, indicating a preferred orientation of the particles perpendicular to the magnetic field.

\section{Optical polarization microscopy}

When the particles are aligned the sample becomes birefringent. The degree of birefringence can be observed conveniently by polarization microscopy. Here we selected the sample orientation in such a way that when the suspension was indeed birefringent, i.e. when the cores were aligned, a maximum of light reached the detector (CCD camera). We then recorded the evolution of the intensity of the transmitted light as a function of temperature. The measurements were somewhat complicated by the presence of multiple scattering of light in these dense suspensions. Multiple light scattering also leads to depolarization of light even in the absence of birefringence. Moreover the increase of refractive index of the particles upon collapse of the shell led to a temperature dependence of the multiple scattering contributions which had to be taken into account.

We therefore characterized the alignment of our particles by comparing the transmission of light for a random sample $\mathrm{I}_{0}(\mathrm{~T})$ and for a sample that had been exposed to a strong magnetic field $\mathrm{I}_{\mathrm{B}}(\mathrm{T})$, Figure $\mathrm{S} 6$.

$$
\Delta I=I_{B}(T)-I_{0}(T)
$$

We used a Leica DMIRB inverted microscope equipped with a temperature controlled stage, in the crossed-polarizer configuration (Figure S6). We then carried out two experiments, one where the particles were oriented prior to the experiment, the other where the orientation of the particles was random. The following routine was applied: in a pretreatment step, the sample was first heated up to $35^{\circ} \mathrm{C}$, exposed to an external magnetic field, then cooled down to $10^{\circ} \mathrm{C}$ in 
Supplementary Material (ESI) for Soft Matter

This journal is (c) The Royal Society of Chemistry 2010

the presence of the field. The magnetic field was then removed and the measurement started. After reaching $35^{\circ} \mathrm{C}$ the sample was left to equilibrate and then cooled down again to $10^{\circ} \mathrm{C}$ without a field.
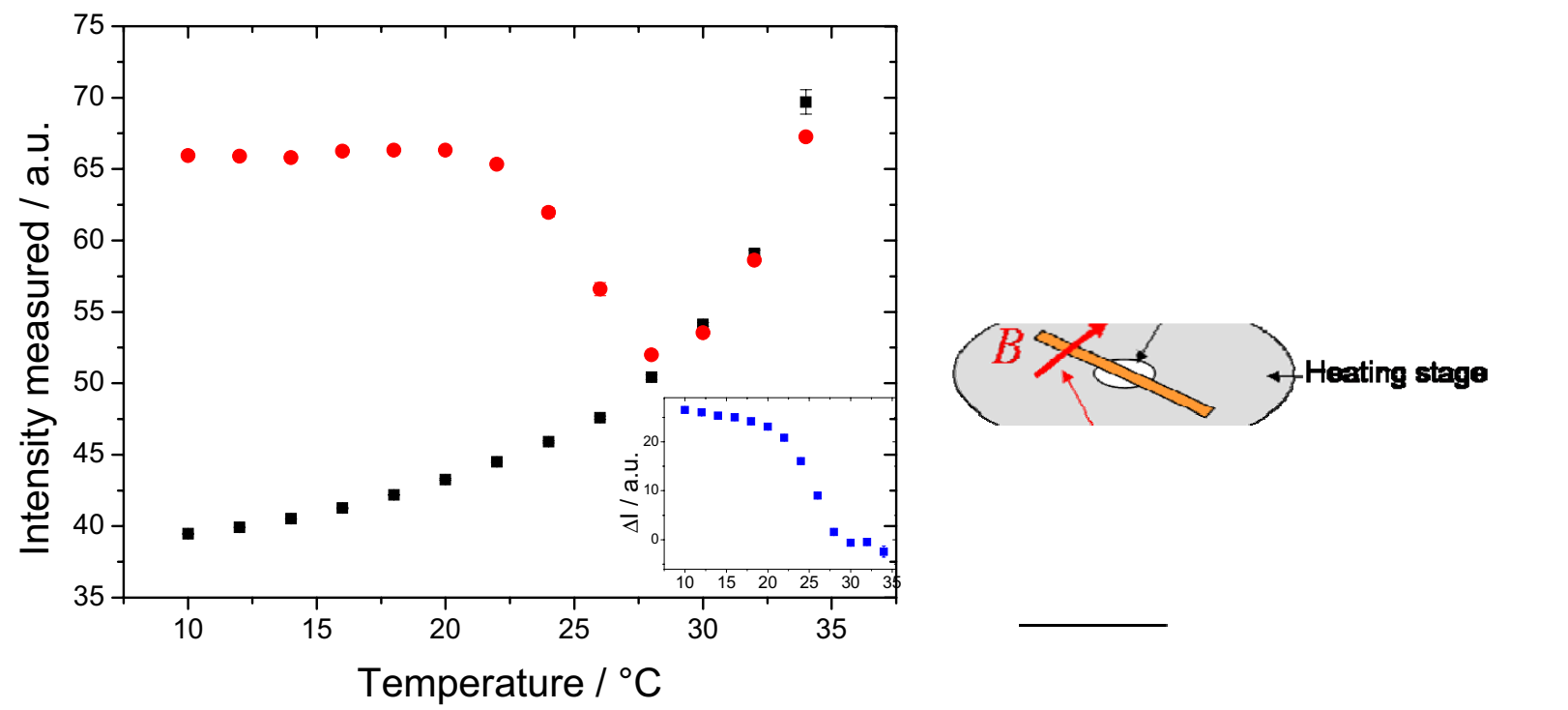

Figure S6. Intensity measured by optical polarization microscopy as a function of temperature. Black squares: without pre-alignment. Red circles: with pre-alignement. Inset: Intensity difference $\Delta \mathrm{I}$. A sketch of the setup is shown on the right-hand side.

\section{References}

1 M. Ocana, M. P. Morales and C. J. Serna, J. Colloid Interface Sci., 1999, 212, 317 ;

2 S. Sacanna, L. Rossi, B. W. M. Kuipers and A. P. Philipse, Langmuir, 2006, 22, 1822; 